HUTP-03/A051

\title{
TeV Symmetry and the Little Hierarchy Problem
}

\author{
Hsin-Chia Cheng and Ian Low \\ Jefferson Physical Laboratory, Harvard University, Cambridge, MA 02138
}

\begin{abstract}
Constraints from precision electroweak measurements reveal no evidence for new physics up to $5-7 \mathrm{TeV}$, whereas naturalness requires new particles at around $1 \mathrm{TeV}$ to address the stability of the electroweak scale. We show that this "little hierarchy problem" can be cured by introducing a symmetry for new particles at the TeV scale. As an example, we construct a little Higgs model with this new symmetry, dubbed $T$-parity, which naturally solves the little hierarchy problem and, at the same time, stabilize the electroweak scale up to $10 \mathrm{TeV}$. The model has many important phenomenological consequences, including consistency with the precision data without any finetuning, a stable weakly-interacting particle as the dark matter candidate, as well as collider signals completely different from existing little Higgs models, but rather similar to the supersymmetric theories with conserved $R$-parity.
\end{abstract}




\section{INTRODUCTION}

Standard Model is very successful in describing all known phenomena in particle physics to date. It is nonetheless theoretically incomplete as the mass-squared parameter for the Higgs doublet receives quadratically divergent corrections at the quantum level and hence is very sensitive to ultraviolate physics. In order for the Higgs mass to be naturally in the $\mathcal{O}(100) \mathrm{GeV}$ range, new physics which couples to the Higgs sector should appear at the scale $\sim 1 \mathrm{TeV}$ or below to cut off the quadratically divergent contributions.

At low energies, new physics can be integrated out and its effects are parametrized in terms of higher dimensional operators involving only Standard Model fields [1]. Precision experimental measurements constrain the sizes of various higher dimensional operators and consequently the scales of the corresponding new physics [2]. The most stringent bounds are on the operators which break the (approximate) symmetries of the Standard Model, such as those violating baryon number, flavor and CP symmetries. New physics which occurs at the TeV scale should respect these Standard Model symmetries in order not to generate any dangerous operator with a significant size. In the low energy effective theory, however, there are operators, generated by the new physics, which conserve baryon number, flavor and CP symmetries. Precision electroweak measurements put strong constraints on many operators of this kind, and so far suggest no evidence for new physics up to $\gtrsim 5-7 \mathrm{TeV}[3]$. This creates some tension with the naturalness requirement, however, which expects new physics at $\sim 1 \mathrm{TeV}$ to cut off the quadratic divergence to the Higgs mass-squared. Indeed, many models which address the stabilization of the electroweak scale have new particles in the $1 \mathrm{TeV}$ range in order to cancel the quadratic divergences incurred by the Standard Model particles. The amount of fine-tuning required to reconcile the difference here is not severe, and one may or may not take this "little hierarchy problem" seriously. Nevertheless, these constraints definitely present an interesting challenge to theorists trying to build models which deal with the stability of the electroweak symmetry breaking scale.

In this paper we consider how the little hierarchy problem can be resolved in a natural way by introducing a new symmetry at the $\mathrm{TeV}$ scale. In deriving the bound of $5-7 \mathrm{TeV}$, it was assumed that these higher dimensional operators are generated at tree level with $\mathcal{O}(1)$ couplings to the Standard Model fields. On the other hand, the cancellation of the quadratic divergences involves quantum loop diagrams only. Thus if one eliminates the tree level exchanges of the new particles among the Standard Model fields, the bound on the scale of the new physics can possibly be lowered by an order of magnitude without spoiling the cancellation of the quadratic divergences, making the existence of the new particles in the $1 \mathrm{TeV}$ range consistent with precision electroweak data. In the next section, we propose a symmetry, acting on the new $\mathrm{TeV}$ scale particles, which achieves the above goal. In Sec. III we present a realistic model, with the aforementioned new symmetry, in the framework of the recently proposed little Higgs theories, which provide a new way to cancel the one-loop quadratic divergences of the Higgs mass-squared and stabilize the electroweak scale [4, 5, 6, 7]. The existence of this new symmetry has many important phenomenological consequences on future collider searches of new physics, as well as dark matter, which will be discussed in Sec. IV] Then we conclude in Sec. D]

During the final stage of this project, Ref. [8] appeared which also pointed out the possibility of imposing a new symmetry at the $\mathrm{TeV}$ scale to lower the scale of new physics while evading constraints from precision measurements. The discussion there parallels ours in Sec. II though the stabilization of the electroweak scale was not addressed in that article. 


\section{NEW SYMMETRY FOR TEV SCALE PARTICLES}

The origin of electroweak symmetry breaking (EWSB) is one of the most prominent questions in particle physics nowadays. If it is indeed triggered by the vacuum expectation value (VEV) of a scalar Higgs doublet, naturalness arguments require new physics at or below $1 \mathrm{TeV}$ to cut off the quadratically divergent contributions to the Higgs mass-squared. On the other hand, if EWSB is caused by some strong dynamics, one also expects that it occurs at the $1 \mathrm{TeV}$ scale in order to obtain the EWSB scale of $246 \mathrm{GeV}$. At the Large Hadron Collider ( $\mathrm{LHC}$ ), the TeV scale physics will be fully explored. It is important to be able to anticipate what kind of signals for new physics may show up in these upcoming experiments.

Current experimental data already give some constraints on possible new physics at the $\mathrm{TeV}$ scale. Absence of nucleon decays and strong bounds on flavor-changing neutral currents indicate that these effects cannot receive any significant contributions from the TeV scale physics, which implies baryon number conservation and approximate flavor symmetries at the TeV scale. Precision electroweak measurements also put constraints on many dimensionsix operators consistent with baryon, flavor and CP symmetries. The scales which suppress these operators are required to be larger than 2-7 TeV, depending on the operators and the Higgs mass, as was discussed in Ref. 3]. Generally speaking these operators arise by exchanging new heavy particles, and the bound on the sizes of the operators translates into the bound on the masses of the new particles and their couplings to the Standard Model fields. If the new particles are responsible for cancelling the quadratic divergences to the Higgs mass-squared, their masses have to be at $\sim 1 \mathrm{TeV}$ by naturalness. One therefore needs to worry about the compatibility of the existence of these particles with the precision electroweak data. Note, however, that the quadratic sensitivity to the high energy physics of the Higgs mass-squared parameter is a result of loop contributions. To cancel the quadratic divergences the new particles at the $\mathrm{TeV}$ scale ${ }^{1}$ only need to contribute to the Higgs mass at the loop level, i.e., we only need interaction vertices involving two or more new TeV particles. On the other hand, generating higher dimensional operators at the tree level requires different interaction vertices, those containing only one new $\mathrm{TeV}$ particle. Therefore, it is possible to suppress the tree level contributions due to the new physics without modifying the cancellation of the loop contributions. The simplest and most natural way to implement this is to have a new symmetry acting on new TeV particles, while all the Standard Model fields are neutral under the new symmetry. Then there can be no interaction vertex involving the Standard Model particles and a single new TeV particle charged under the symmetry. The interactions containing more than one $\mathrm{TeV}$ particles, on the other hand, can still be allowed. Of course, not every $\mathrm{TeV}$ scale particle would induce large higher dimensional operators which affect the precision electroweak measurements, so in practice we only need the dangerous particles, for example $W^{\prime}$ and $Z^{\prime}$, to be charged under this symmetry. The simplest choice for the new symmetry is just a $Z_{2}$ parity, but larger symmetry groups are also possible. With the new symmetry, higher dimensional operators are generated only at the loop level, and new particles as light as a few hundred $\mathrm{GeV}$ can be perfectly consistent with the precision electroweak data.

There are existing models with such symmetry acting only on the new particles. The

\footnotetext{
${ }^{1}$ The new particles can be much lighter than $1 \mathrm{TeV}$. For simplicity we will simply call all these new particles at or below the $\mathrm{TeV}$ scale $\mathrm{TeV}$ particles.
} 
most popular and well-known example is the Minimal Supersymmetric Standard Model (MSSM) with $R$-parity conservation. In MSSM, all Standard Model particles have positive $R$-parity and all superpartners have negative $R$-parity. Superpartner loops cancel the quadratic divergences from the Standard Model particle loops, but in the low energies there is no higher dimensional operator induced by superpartners at the tree level. For a large portion of the parameter space, MSSM is consistent with all the precision data. This is one of the major reasons which make the MSSM the leading candidate for physics beyond the Standard Model. On the other hand, without $R$-parity, there are many strong constraints on the $R$-parity violating couplings which require them to be unnaturally small. Although supersymmetry is aesthetically appealing, $R$-parity is the reality check that ensures the consistency of supersymmetric models with precision experiments.

Another closely related example is the KK-parity in the Universal Extra Dimensions (UEDs), where all Standard Model particles propagate in some number of compactified extra dimensions [9, 10, 11, 12]. The compactification breaks the translational invariance in the extra dimensions down to some discrete subgroup corresponding to the geometrical symmetry of the compactified space. As a result, the momentum conservation in extra dimensions is reduced to the KK-parity conservation of the Kaluza-Klein (KK) states of the Standard Model fields. The KK-parity prohibits the lowest KK states from contributing to the higher dimensional operators at the tree level, therefore allowing them to be as light as $300 \mathrm{GeV}$ [10, 13]. The contributions from higher KK states may also be suppressed if the mixing with the zero mode is small. Although the simplest UED scenario, where the KK state loops do not cancel the quadratic divergence of the Higgs mass-squared, does not directly address the little hierarchy problem, the KK-parity allows the sizes of the extra dimensions to be large enough to be probed in the near future. This feature makes the UED model very interesting phenomenologically. In contrast, extra-dimensional models without KK parity have much stronger bounds on the masses of the KK states, and hence the sizes of the extra dimensions [14], which makes these models beyond direct probe of near future experiments.

The above discussion suggests that this new TeV symmetry could be a key to the phenomenological success of a model with new particles at or below $1 \mathrm{TeV}$ scale, especially one concerning the stabilization of the electroweak scale. Recently a new class of theories, inspired by the dimensional deconstruction [15, 16] and dubbed little Higgs theories, was proposed to address the stability of the electroweak scale in a new way [4, 5, 6, 7]. In these theories, the quadratic divergence of the Standard Model loops are cancelled at one loop by new states, with the same spin as the Standard Model particles, appearing at the TeV scale. The cut-off scale of the little Higgs theories can be as high as $10 \mathrm{TeV}$ or above while at the same time stabilizing the electroweak scale without fine-tuning. There are a number of variations of the little Higgs model [17, 18, 19, 20, 21, 22], but in all cases so far the new $\mathrm{TeV}$ particles couple directly to the Standard Model particles and one needs to worry about the impact on the precision electroweak physics from these new particles. In the next section we will show that it is possible to construct a little Higgs model with a new parity at the TeV scale such that all the Standard Model particles are neutral under the new symmetry. This model therefore solves the little hierarchy problem naturally and is in good agreement with the precision electroweak measurements. 


\section{A LITTLE HIGGS MODEL}

Little Higgs theories provide a new way to stabilize the electroweak scale. They revive an old idea of the Higgs being a pseudo-Nambu-Goldstone boson (PNGB) [23, 24, 25, 26, [27, 28, 29]. A such model is based on a chiral Lagrangian in which a global symmetry is both spontaneously broken and explicitly broken by some weakly-interacting couplings. The crucial new ingredient for little Higgs model is that each coupling preserves a subset of the global symmetry under which the Higgs doublet (little Higgs) is an exact Nambu-Goldstone boson. The little Higgs only learns its PNGB nature in the presence of more than one set of couplings. Therefore, there is no one-loop quadratic divergence to the little Higgs mass-squared. Any correction to the Higgs mass-squared is suppressed by two loop factor relative to the cutoff, raising the cutoff to $\sim 10 \mathrm{TeV}$ without destabilizing the electroweak scale. A number of models based on various symmetry groups have been constructed. A universal feature is that there exist new gauge bosons, fermions, and scalars at the TeV scale which cancel the one-loop quadratic divergence to the Higgs mass-squared from the Standard Model electroweak gauge bosons, top quark, and the Higgs quartic coupling, respectively. The corrections to the electroweak observables for several models have been computed in Refs. 30, 31, 32, 33]. In general there are strong constraints on the viable parameter space, even though they are quite model dependent. The largest corrections often come from the new gauge boson exchanges and VEVs of the $S U(2)_{W}$ triplet scalars. It is possible to find models, with acceptable fine-tunings, for which such constraints are loosened in some region of parameter space. This in turn suggests that the tree-level corrections to the electroweak observables are not an essential part of the little Higgs models. It is therefore interesting to find models in which these tree-level contributions are absent for symmetry reasons.

A natural starting point for model building is to consider moose type models based on deconstruction. They often contain some geometric symmetries which may be used for our purpose. For example, leaving out fermions for now, the minimal moose model in Ref. [6] has a reflection symmetry which exchanges the two sites if the same $S U(2) \times U(1)$ subgroup is gauged on each site with equal gauge coupling. However, the way the Standard Model chiral fermions were introduced there breaks this symmetry, and one needs a way to distribute the Standard Model fermions evenly between the two sites. This can be done by putting one more site in the model and placing mirror fermions on the extra site, as will be discussed in detail later in this section. Another important issue is that because the non-linear sigma model is getting strongly coupled at the $\sim 10 \mathrm{TeV}$ scale, certain operators generated at that scale may be enhanced by the strong couplings, and hence violate the bounds from the electroweak precision measurements. In particular, the dimension-six operator involving the Higgs field, $\left(h^{\dagger} D_{\mu} h\right)^{2}$, may be generated with a coefficient $\sim 1 / f^{2} \approx(4 \pi / \Lambda)^{2}$, where $f \sim 1$ $\mathrm{TeV}$ is the symmetry breaking scale and $\Lambda$ is the cutoff. This operator arises from expanding the non-linear chiral Lagrangian, breaks the custodial $S U(2)_{C}$ symmetry, and contributes to the $\rho$ parameter. A simple way to avoid this is to choose the global symmetry to contain an $S U(2)_{C}$ symmetry, eliminating such an operator from the non-linear chiral Lagrangian.

In the following we construct a little Higgs model with a $Z_{2}$ symmetry acting on the TeV scale new particles. For simplicity, we will call it "T-parity," although some of the new particles may be lighter than $1 \mathrm{TeV}$ and there may still be a few $\mathrm{TeV}$ particles even under the parity. The $T$-parity arises due to a geometric reflection symmetry of our theory space, which consists of three sites and five links. At each site $G_{i}, i=a, b, c$, there is an $S O(5)$ global symmetry in which an $S U(2) \times U(1)$ subgroup is gauged. The reflection symmetry 


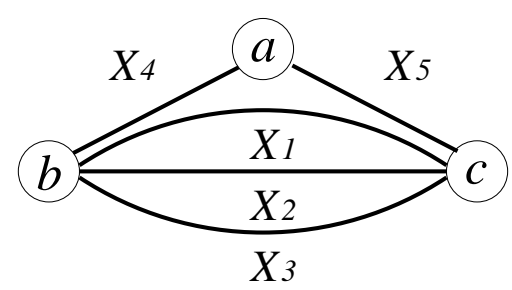

FIG. 1: The moose diagram for the theory space. It has the topology of a torus.

ensures the gauge couplings on sites $b$ and $c$ are equal. The five link fields $X_{j}=\exp \left(i x_{j} / f\right)$, $j=1, \cdots, 5$, are the non-linear sigma model fields associated with the theory space, as indicated in Fig. 1. This theory space is a variation of the minimal moose model in Ref. [6], with one additional site inserted in one of the links. However, we have chosen the global symmetry to be $S O(5)$ in order to have custodial $S U(2)_{C}$ as an approximate symmetry [20]. It has a large, approximate $[S O(5)]^{10}$ global symmetry spontaneously broken to $[S O(5)]^{5}$. The cutoff of the non-linear sigma model is taken to be $\Lambda \sim 4 \pi f \sim 10 \mathrm{TeV}$. Below this cutoff the effective theory is described by the Lagrangian

$$
\mathcal{L}=\mathcal{L}_{G}+\mathcal{L}_{X}+\mathcal{L}_{\psi},
$$

where $\mathcal{L}_{G}$ includes the kinetic terms for the $X_{j}$ as well as the gauge interactions, $\mathcal{L}_{X}$ contains various plaquette operators for the non-linear sigma model fields, and $\mathcal{L}_{\psi}$ involves interactions with fermions. We now describe these three sectors in turn.

In the gauge sector, the $[S U(2) \times U(1)]^{3}$ gauge symmetry is spontaneously broken to the diagonal subgroup, which is taken to be the electroweak $S U(2)_{W} \times U(1)_{Y}$. Thus there are two sets of heavy gauge bosons in the low energy effective theory, which can be taken to be

$$
\begin{array}{ll}
A_{\mu}^{A} \sim\left(g_{b} A_{\mu}^{(b)}-g_{c} A_{\mu}^{(c)}\right), & A_{\mu}^{S} \sim\left(g_{b} A_{\mu}^{(b)}+g_{c} A_{\mu}^{(c)}-2 g_{a} A_{\mu}^{(a)}\right), \\
B_{\mu}^{A} \sim\left(g_{b}^{\prime} B_{\mu}^{(b)}-g_{c}^{\prime} B_{\mu}^{(c)}\right), & B_{\mu}^{S} \sim\left(g_{b}^{\prime} B_{\mu}^{(b)}+g_{c}^{\prime} B_{\mu}^{(c)}-2 g_{a}^{\prime} B_{\mu}^{(a)}\right),
\end{array}
$$

where $A_{\mu}^{(i)}, B_{\mu}^{(i)}$ are the $S U(2) \times U(1)$ gauge bosons at the $i$ th site and $g_{i}, g_{i}^{\prime}$ are the corresponding gauge couplings. We have chosen these particular combinations because they have definite parity under the reflection symmetry of the theory space. $A_{\mu}^{S}$ and $B_{\mu}^{S}$ are even under the interchange of sites $b$ and $c$, whereas $A_{\mu}^{A}$ and $B_{\mu}^{A}$ are odd; this defines the $T$-parity of the heavy gauge bosons. Ordinarily these heavy gauge bosons have masses of the order $g f$, which is around $1 \mathrm{TeV}$ if gauge couplings are $\mathcal{O}(1)$. However, we would like to decouple the $T$-even heavy gauge bosons by taking the gauge group at site $a$ to be strongly coupled: $g_{a} \sim 4 \pi$. Then the $T$-even heavy gauge bosons will be massive in the order of $10 \mathrm{TeV}$, and are consisted of mostly site $a$ gauge bosons. This suppresses the tree-level contributions to the electroweak observables from the $T$-even heavy gauge bosons, if the Standard Model fields live away from site $a$. On the other hand, the little Higgses remain light as they still require gauge couplings on sites $b$ and $c$, both $\mathcal{O}(1)$, to know that they are not exact NGBs.

The embedding of the $S U(2) \times U(1)$ gauge group in the global $S O(5)$ is the same as in Ref. [20], where the $S O(5)$ generators are labeled as $T^{l}, T^{r}$, and $T^{v}$ for the $S U(2)_{l}, S U(2)_{r}$, and $S O(5) / S O(4)$ generators respectively, using the $T^{l a}$ generators for $S U(2)$ and $T^{r 3}$ for $U(1)$. It is more convenient, when we introduce fermions later, to use the language of $S p(4)$, which is the universal covering group of $S O(5)$. Throughout the paper we will use the bi-spinor notation for the link fields which parameterize the coset space of $(S p(4) \times$ 
$S p(4)) / S p(4)$. The $S U(2)_{l}$ and $S U(2)_{r}$ simply act on the upper and lower two components, respectively, of the fundamental representation of $S p(4)$. In the scalar sector each link field $X_{j}$ contains, under the $S U(2)_{W}$ gauge group, a triplet, three singlets, and a complex doublet. The plaquette operators we include in the Lagrangian are

$$
\begin{aligned}
& \mathcal{L}_{X}= \mathcal{P}_{T S}+\mathcal{P}_{D S}+\mathcal{P}_{D}+\mathcal{P}_{Q}+\mathcal{P}_{T^{3}}, \\
& \mathcal{P}_{T S}=\tau f^{4} {\left[\operatorname{Tr}\left(\Omega X_{1} X_{3}^{\dagger} \Omega X_{1} X_{3}^{\dagger}\right)+\operatorname{Tr}\left(\Omega X_{1}^{\dagger} X_{3} \Omega X_{1}^{\dagger} X_{3}\right)\right] } \\
&+\tau^{\prime} f^{4}\left[\operatorname{Tr}\left(\Omega X_{2} X_{3}^{\dagger} \Omega X_{2} X_{3}^{\dagger}\right)+\operatorname{Tr}\left(\Omega X_{2}^{\dagger} X_{3} \Omega X_{2}^{\dagger} X_{3}\right)\right]+\text { h.c. }, \\
& \mathcal{P}_{D S}=\sigma f^{4}\left[\operatorname{Tr}\left(\Omega^{\prime} X_{4} \Omega^{\prime} X_{4}^{\dagger}\right)+\operatorname{Tr}\left(\Omega^{\prime} X_{5} \Omega^{\prime} X_{5}^{\dagger}\right)\right]+\text { h.c. }, \\
& \mathcal{P}_{D}=\omega f^{4}\left[\operatorname{Tr}\left(\Omega X_{4} \Omega X_{4}^{\dagger}\right)+\operatorname{Tr}\left(\Omega X_{5} \Omega X_{5}^{\dagger}\right)\right]+\text { h.c. }, \\
& \mathcal{P}_{Q}=\lambda f^{4}\left[\operatorname{Tr}\left(X_{1} X_{2}^{\dagger} X_{3} X_{5}^{\dagger} X_{4}^{\dagger}\right)+\operatorname{Tr}\left(X_{1}^{\dagger} X_{2} X_{3}^{\dagger} X_{4} X_{5}\right)\right]+\text { h.c. }, \\
& \mathcal{P}_{T^{3}=}=i \epsilon f^{4} \operatorname{Tr} T^{r 3}\left(X_{1} X_{2}^{\dagger} X_{3} X_{5}^{\dagger} X_{4}^{\dagger}+X_{1}^{\dagger} X_{2} X_{3}^{\dagger} X_{4} X_{5}\right. \\
&\left.\quad+X_{3} X_{5}^{\dagger} X_{4}^{\dagger} X_{1} X_{2}^{\dagger}+X_{3}^{\dagger} X_{4} X_{5} X_{1}^{\dagger} X_{2}\right)+ \text { h.c. },
\end{aligned}
$$

where $\Omega=\operatorname{diag}(-1,-1,1,1)$ and $\Omega^{\prime}=\operatorname{diag}(-1,-1,-1,1)$. Since we only gauge an $S U(2) \times$ $U(1)$ subgroup at each site, only two triplets and two singlets are eaten, giving masses to the broken gauge bosons. The remaining three triplets and thirteen singlets, as well as three doublets, obtain masses of order $1 \mathrm{TeV}$ from plaquette operators $\mathcal{P}_{T S}, \mathcal{P}_{D S}, \mathcal{P}_{D}$, and $\mathcal{P}_{Q}$. Note that a plaquette operator of the type $\Omega X \Omega X^{\dagger}$ gives mass only to scalars sitting in the off-diagonal blocks in $X$, whereas $\Omega X \Omega X$ gives mass only to the diagonal blocks [17]. More specifically, the number of scalars becoming massive through these plaquette operators is as follows: two triplets and six singlets from $\mathcal{P}_{T S}$, two doublets and four singlets from $\mathcal{P}_{D S}$, and one doublet, one triplet and three singlets from $\mathcal{P}_{Q} \cdot{ }^{2}$ Only two electroweak doublets remain light. Therefore in the low energies our construction gives rise to a two Higgs doublets model. Quartic interactions of the Higgs doublets come from the plaquette $\mathcal{P}_{Q}$, which can be analyzed using the method in Ref. 17], or simply by expanding the plaquette operators and setting all the heavy fields to zero. It is hardly surprising that the Higgses have the same quartic potential as in the $S O(5)$ minimal moose model in Ref. 20]. Moreover, the $T^{r 3}$ plaquettes $\mathcal{P}_{T^{3}}$ provide a Higgs mass term $i h_{1} h_{2}^{\dagger}$, which is necessary to have stable electroweak symmetry breaking, and its coefficient $\epsilon$ is set to be a loop factor less than $\lambda$ in $\mathcal{P}_{Q}[20]$. All other coefficients in $\mathcal{L}_{X}$ are $\mathcal{O}(1)$.

The plaquette operators in Eq. (4i) are invariant under the reflection symmetry $P$ of the theory space

$$
\begin{aligned}
& X_{j} \leftrightarrow X_{j}^{\dagger}, \quad j=1,2,3 \\
& X_{4} \leftrightarrow X_{5}^{\dagger},
\end{aligned}
$$

\footnotetext{
${ }^{2}$ The $\mathcal{P}_{D}$ plaquette gives mass to the same two doublets as $\mathcal{P}_{D S}$ does, and will be generated by fermion interactions discussed later.
} 
and the Goldstone fields transform as

$$
\begin{aligned}
& x_{j} \leftrightarrow-x_{j}, \quad j=1,2,3 \\
& x_{4} \leftrightarrow-x_{5} .
\end{aligned}
$$

Therefore only one linear combination of Goldstone bosons $x_{4}-x_{5}$ is even under $P$, whose triplet and one of the singlets are eaten by the even gauge bosons $A_{\mu}^{S}$ and $B_{\mu}^{S}$, while the rest are all odd under the reflection $P$. This reflection symmetry $P$ is broken once the light Higgses develop VEVs to break the electroweak symmetry. However, there is still a $Z_{2}$ parity which remains unbroken. To see this, we make use of the fact that, by multiplying $\Omega$ to the link fields on both sides, a generic Goldstone field,

$$
X=\exp (i x)=\exp i\left(\begin{array}{cc}
\phi & h \\
h^{\dagger} & s
\end{array}\right),
$$

transforms as

$$
\Omega X \Omega=\exp (i \Omega x \Omega)=\exp i\left(\begin{array}{cc}
\phi & -h \\
-h^{\dagger} & s
\end{array}\right),
$$

where the triplet $\phi$ and singlets $s$ sit in the upper left and lower right $2 \times 2$ blocks, respectively, and the doublet $h$ sits in the off-diagonal blocks transforming as $(\mathbf{2}, \mathbf{2})$ under the $S U(2)_{l} \times$ $S U(2)_{r}$ subgroup. One can check that the plaquette operators in Eq. (44) are invariant under the combined operation $T=P \Omega$,

$$
\begin{aligned}
& X_{j} \leftrightarrow \Omega X_{j}^{\dagger} \Omega, \quad j=1,2,3 \\
& X_{4} \leftrightarrow \Omega X_{5}^{\dagger} \Omega .
\end{aligned}
$$

Both light doublets, as well as the two heavy doublets, are even under the combined operation $P \Omega$, which we take as the definition of $T$-parity for scalar particles, whereas all the heavy triplets and all but two singlets are odd. The $T$-parity remains unbroken even after the light Higgs doublets acquire VEVs.

For the fermion sector, the reflection symmetry of the theory space forces identical fermion contents at sites $b$ and $c$. Therefore we need to spread out the standard model fermions evenly between those two sites. ${ }^{3}$ We do this by introducing additional mirror Weyl fermions at site $a$ and coupling them through link fields to fermions at sites $b$ and $c$, of which a linear combination marries the mirror fermion to become massive in the order of $1 \mathrm{TeV}$. The orthogonal combination remains massless and are taken to be the Standard Model fermions. Thus it is necessary to introduce a copy of Standard Model fermion content at each site $b$ and $c$, and a copy of mirror Standard Model fermions at site $a$. Notice, however, that the $U(1)$ charge assignments for all these fermions can be different from the physical Standard Model fermions. There is some freedom in the $U(1)$ charge assignments as the fermions may be charged under more than one $U(1)$ 's, as long as they have the correct hypercharges under the unbroken diagonal $U(1)$. In order to avoid large tree-level couplings between the Standard Model fermions and the $U(1)_{a}$ gauge boson, we require that the fermions at site $b$ and $c$ to be neutral under $U(1)_{a}$. A convenient choice for the $U(1)$ charges, which makes

\footnotetext{
${ }^{3}$ The gauge group at site $a$ is strongly coupled and we demand the Standard Model fermions to be neutral under $[S U(2) \times U(1)]_{a}$.
} 
TABLE I: The U(1) charge assignments for fermions. All fermions in the table are left-handed. We denote the $S U(2)$ doublet quarks and leptons by $q$ and $\ell, S U(2)$ singlet quarks and leptons by $u^{c}, d^{c}, e^{c}$, and $\nu^{c}$. The $U(1)$ charges for fermions at site $c$ are simply those of fermions at site $b$ with $U(1)_{b}$ and $U(1)_{c}$ charges interchanged, as required by the reflection symmetry. The physical $U(1)_{Y}$ hypercharge is the sum of the $U(1)$ charges at all three sites.

\begin{tabular}{|c|c|c|c|c|c|c|c|c|c|c|c|c|}
\hline & $\bar{q}^{(a)}$ & $q^{(b)}$ & $\bar{\ell}^{(a)}$ & $\ell^{(b)}$ & $\bar{u}^{c(a)}$ & $u^{c(b)}$ & $\bar{d}^{c}(a)$ & $d^{c(b)}$ & $\bar{e}^{c(a)}$ & $e^{c(b)}$ & $\bar{\nu}^{c(a)}$ & $\nu^{c(b)}$ \\
\hline$U(1)_{a}$ & 0 & 0 & 0 & 0 & $\frac{1}{2}$ & 0 & $-\frac{1}{2}$ & 0 & $-\frac{1}{2}$ & 0 & $\frac{1}{2}$ & 0 \\
\hline$U(1)_{b}$ & $-\frac{1}{12}$ & $\frac{1}{12}$ & $\frac{1}{4}$ & $-\frac{1}{4}$ & $\frac{1}{12}$ & $-\frac{7}{12}$ & $\frac{1}{12}$ & $\frac{5}{12}$ & $-\frac{1}{4}$ & $\frac{3}{4}$ & $-\frac{1}{4}$ & $-\frac{1}{4}$ \\
\hline$U(1)_{c}$ & $-\frac{1}{12}$ & $\frac{1}{12}$ & $\frac{1}{4}$ & $-\frac{1}{4}$ & $\frac{1}{12}$ & $-\frac{1}{12}$ & $\frac{1}{12}$ & $-\frac{1}{12}$ & $-\frac{1}{4}$ & $\frac{1}{4}$ & $-\frac{1}{4}$ & $\frac{1}{4}$ \\
\hline
\end{tabular}

the fermion mass terms we are about to write down gauge invariant, is described in Table【. Given that the $U(1)$ charge assignments are rather odd looking at first glance, it is quite interesting to check that all the anomalies cancel if one includes right-handed neutrinos.

Through link fields $X_{4}$ and $X_{5}$, the fermions at site $a$ marry with a linear combination of fermions at sites $b$ and $c$ and become massive. To simplify notations, let us define

$$
\tilde{X}_{i}=X_{i}+\Omega X_{i} \Omega, \quad i=4,5,
$$

which contain only the diagonal $2 \times 2$ blocks of $X_{4}$ and $X_{5}$, and group the fermions as follows

$$
\begin{array}{ll}
\bar{Q}^{(a)}=\left(\bar{q}^{(a)}, \bar{d}^{c(a)}, \bar{u}^{c(a)}\right), & \bar{L}^{(a)}=\left(\bar{\ell}^{(a)}, \bar{e}^{c(a)}, \bar{\nu}^{c(a)}\right), \\
Q^{(j)}=\left(q^{(j)}, d^{c(j)}, u^{c(j)}\right)^{T}, & L^{(j)}=\left(\ell^{(j)}, e^{c(j)}, \nu^{c(j)}\right)^{T}, \quad j=b, c .
\end{array}
$$

Then, the masses of the $\mathrm{TeV}$ fermions can come from the interactions

$$
\mathcal{L}_{f m}=\kappa_{q} f \bar{Q}^{(a)}\left(\tilde{X}_{4}^{\dagger} Q^{(b)}+\tilde{X}_{5} Q^{(c)}\right)+\kappa_{\ell} f \bar{L}^{(a)}\left(\tilde{X}_{4}^{\dagger} L^{(b)}+\tilde{X}_{5} L^{(c)}\right) .
$$

Given the charge assignments in Table I. Eq. (17) is invariant under gauge transformations at every site. At first order in $f, \mathcal{L}_{f m}$ gives rise to a Dirac mass term

$$
\kappa_{q} f \bar{Q}^{(a)}\left(Q^{(b)}+Q^{(c)}\right)+\kappa_{\ell} f \bar{L}^{(a)}\left(L^{(b)}+L^{(c)}\right) .
$$

These heavy fermions have masses at around $1 \mathrm{TeV}$. We shall assume that their masses are approximately flavor universal by some flavor symmetry so that they do not induce large flavor changing effects. The massless linear combinations $\left(Q^{(b)}-Q^{(c)}\right) / \sqrt{2}$ and $\left(L^{(b)}-\right.$ $\left.L^{(c)}\right) / \sqrt{2}$ become the Standard Model fermions in the low energies. The interactions in Eq. (17) also induce the plaquette operators $\mathcal{P}_{D}$, which lift extra scalar doublets, through loops. ${ }^{4}$

For Yukawa couplings, we first concentrate on the top sector, which must be dealt with in a way without introducing quadratic divergences to the Higgs mass. Toward this end we

${ }^{4} \mathcal{P}_{D S}$ can also be generated radiatively if we do not include right-handed neutrinos or they have different couplings from charged leptons. 
introduce additional vector-like colored fermions $\psi_{u, d}^{(j)}, \psi_{u, d}^{c(j)}, j=b, c$, with $\psi_{u}^{(j)}\left(\psi_{d}^{(j)}\right)$ having the opposite $U(1)$ charges of $u^{c(j)}\left(d^{c(j)}\right)$. Defining

$$
\mathcal{Q}^{(j)}=\left(q_{3}^{(j)}, \psi_{u}^{(j)}, \psi_{d}^{(j)}\right)^{T}, \quad \mathcal{U}^{c(j)}=\left(0_{2}, u_{3}^{c(j)}, 0\right), \quad j=b, c \quad,
$$

the top Yukawa coupling is then generated by

$$
\begin{aligned}
\mathcal{L}_{\text {top }}= & y_{1} f\left(\mathcal{U}^{c(b)} X_{1} X_{3}^{\dagger} \mathcal{Q}^{(b)}+\mathcal{U}^{c(c)} \Omega X_{1}^{\dagger} X_{3} \Omega \mathcal{Q}^{(c)}\right) \\
& +f \sum_{j=b, c}\left(y_{2} \psi_{u}^{(j)} \psi_{u}^{c(j)}+y_{3} \psi_{d}^{(j)} \psi_{d}^{c(j)}\right) .
\end{aligned}
$$

There is only one massless component left at this stage, which we take to be the physical top quark,

$$
t=\frac{1}{\sqrt{2\left(y_{1}^{2}+y_{2}^{2}\right)}}\left[y_{1}\left(u_{3}^{(b)}-u_{3}^{(c)}\right)+y_{2}\left(\psi_{u}^{(b)}-\psi_{u}^{(c)}\right)\right],
$$

and the top Yukawa coupling turns out to be

$$
\lambda_{t} \sim \frac{y_{1} y_{2}}{\sqrt{y_{1}^{2}+y_{2}^{2}}}
$$

For the other Yukawa couplings we can simply write down operators in the first line in Eq. (20) without introducing additional vector-like fermions, $\psi_{u}$ and $\psi_{d}$, in Eq. (19). These other Yukawa couplings introduce one-loop quadratic divergences without destabilizing the electroweak scale, since the divergences are suppressed by the smallness of the Yukawa couplings.

The $T$-parity of the fermion is defined as $T=(-1)^{F} P$, where $F$ counts the fermion number and $P$ is the reflection that interchanges sites $b$ and $c$. The reason for the extra minus sign from $(-1)^{F}$ is because fermions at site $a$, even under the reflection $P$, should be $T$-odd since it becomes heavy through the Dirac mass term Eq. (18). This minus sign in turn gives even T-parity for the Standard Model fermions, as desired. Together with $T=\Omega P$ for the scalars, the interactions in $\mathcal{L}_{f m}$ and $\mathcal{L}_{\text {top }}$ are invariant under the $T$-parity, which explains the insertion of the $\Omega$ operators in those interactions. Moreover, the linear combination that becomes massive in the TeV range in Eq. (18) is odd, whereas the massless combination, which becomes the Standard Model fermion in the low energies, is even. All the heavy fermions, except for the following two combinations ${ }^{5}$

$$
\begin{aligned}
t^{\prime} & =\frac{1}{\sqrt{2\left(y_{1}^{2}+y_{2}^{2}\right)}}\left[y_{2}\left(u_{3}^{(b)}-u_{3}^{(c)}\right)-y_{1}\left(\psi_{u}^{(b)}-\psi_{u}^{(c)}\right)\right], \\
\tilde{d}^{\prime} & =\frac{1}{\sqrt{2}}\left(\psi_{d}^{(b)}-\psi_{d}^{(c)}\right),
\end{aligned}
$$

are odd under the $T$-parity. To summarize, we define the $T$-parity as

$$
\begin{array}{ll}
T=P, & \text { for vector bosons, } \\
T=\Omega P, & \text { for scalars, } \\
T=(-1)^{F} P, & \text { for fermions, }
\end{array}
$$

\footnotetext{
${ }^{5}$ Note that the mass of the $\psi_{d}$ field can be lifted to $4 \pi f \sim 10 \mathrm{TeV}$ without spoiling naturalness, due to an accidental $S U(3)$ symmetry discussed in Ref. 20].
} 
and it is a symmetry of our Lagrangian and remains unbroken after electroweak symmetry breaking.

As mentioned earlier, our theory space is a modification of the two-site, minimal $S O(5)$ moose model, whose precision electroweak physics was analyzed in some details in Ref. [20]. There it was shown that the most dangerous contributions are from those due to tree-level heavy gauge boson exchanges, including modification of electroweak currents, four fermion operators, and custodial $S U(2)_{C}$ breaking. The custodial $S U(2)_{C}$ is a good symmetry of the non-linear chiral Lagrangian, but is spontaneously broken by the misalignment of the two Higgs VEVs as a result of the commutator type quartic Higgs coupling. The breaking of $S U(2)_{C}$ then shows up in the $\mathrm{TeV}$ gauge boson couplings [20]. In our model, the $S U(2)_{C}$ is also spontaneously broken by the Higgs VEVs, but the TeV gauge bosons are odd under $T$-parity and do not contribute at tree level. The $T$-even heavy gauge bosons are in the 10 $\mathrm{TeV}$ range and have small couplings to the Standard Model fermions and Higgses, who live away from site $a$. So the contributions due to the $T$-even heavy gauge bosons are also very small. The $S U(2)_{W}$ triplet scalars are all odd under $T$-parity and therefore do not obtain VEVs at all, since the tadpole term is forbidden by $T$-parity. There are several $T$-even scalar doublets and singlets at the $\mathrm{TeV}$ scale, but their tree-level contributions are either suppressed by the small Yukawa couplings of light Standard Model fermions or, in the case for top quark, weakly constrained due to lack of precision data. The leading contributions to the electroweak observables in this model come from loops of the two Higgs doublets and top partners. These contributions were also discussed in Ref. 20] and in general are safe for a wide range of model parameters.

Finally, before concluding this section, one may ask since the site- $a$ gauge bosons are very heavy, one should be able to integrate them out and obtain an effective two-site model at low energies. ${ }^{6}$ The question is then how the fermion interactions preserve the $Z_{2}$ parity in this two-site effective theory. To this end we note that with just sites $b, c$ and links between them, the object

$$
X^{\dagger} D_{\mu}^{(b)} X=X^{\dagger}\left(\partial_{\mu}+i g_{b} A_{\mu}^{(b)}\right) X,
$$

which is invariant under gauge transformations at site $b$, transforms in the same way as $i g_{c} A_{\mu}^{(c)}$. Therefore, we can write down the following gauge invariant interaction for the fermion living on site $c$,

$$
\bar{\psi} i \bar{\sigma}^{\mu}\left[\partial_{\mu}+r i g_{c} A_{\mu}^{(c)}+(1-r) X^{\dagger}\left(D_{\mu}^{(b)} X\right)\right] \psi .
$$

For $g_{b}=g_{c}$ and $r=1 / 2$, the fermion just couples to the massless even combination of the gauge fields. The coupling of a light fermion, a heavy odd fermion, and the odd gauge bosons can also be reproduced by the interaction

$$
\bar{\psi}_{\text {light }} \frac{i \bar{\sigma}^{\mu}}{2}\left[i g_{c} A_{\mu}^{(c)}-X^{\dagger}\left(D_{\mu}^{(b)} X\right)\right] \psi_{\text {heavy }}+\text { h.c. . }
$$

The exact $Z_{2}$ symmetry is not transparent in this language. Nevertheless, it may serve as a useful guide to construct other type of little Higgs models with the $T$-parity.

\footnotetext{
${ }^{6}$ We are indebted to Nima Arkani-Hamed for inspiring conversations on this issue.
} 


\section{PHENOMENOLOGICAL CONSEQUENCES}

To be consistent with the electroweak data, the new TeV particle symmetry can be just an approximate symmetry. However, it is well motivated to keep this symmetry exact. In this case, there are many interesting phenomenological consequences, so we will concentrate on the case of an exact symmetry in this section.

Since all Standard Model particles are neutral under the new TeV symmetry, the lightest particle which transforms non-trivially under this symmetry will be stable. Here we call it the LTP, the lightest T-odd particle. This new symmetry has important implications for future collider experiments, as new particles charged under it cannot be singly produced. Direct searches have to rely on pair-productions. In addition, after they are produced, they will decay to the LTP which is stable. If the LTP is electrically charged, it will give rise to charged tracks in the detector which are easy to identify. However, a charged LTP is not favored as it causes cosmological problems. On the other hand, the neutral LTP will escape the detector, resulting in missing energy signals. Most of the collider phenomenology studies for little Higgs theories so far do not assume this new $\mathrm{TeV}$ symmetry, and the Standard Model fermions can interact directly with a single $\mathrm{TeV}$ gauge boson [34, 35, 36]. Similar to the usual $W^{\prime}$ and $Z^{\prime}$ searches, these studies rely on single $\mathrm{TeV}$ gauge boson productions; neither is there a new stable particle in the decay products. Hence the existence of this new $\mathrm{TeV}$ symmetry escapes conclusions from these previous studies, except Ref. [37] which studies loop induced processes, and dramatically alters the collider phenomenology.

In fact, the collider phenomenology with this new $\mathrm{TeV}$ symmetry is similar to that of the $R$-parity conserving supersymmetric theories (and KK-parity conserving UEDs). The typical signals are jets and/or leptons plus missing energies from decays of heavy new particles with odd $T$-parity. To distinguish it from supersymmetry, we need to know the spins of these new particles which probably requires a lepton collider or extra efforts for a hadron collider. Unlike supersymmetric models, new particles in a little Higgs theory have the same spins as the corresponding Standard Model particles whose quadratic divergences they are supposed to cancel. On the other hand, in UEDs the KK excitations also have the same spins as the Standard Model particles. The difference between our little Higgs model and UEDs is that there is no reason for all the new TeV particles to be closely degenerate in our model. Thus the jets and leptons from decays of the $\mathrm{TeV}$ particles in general will not be soft in the little Higgs model, unlike in the UEDs, which makes their detection easier. Moreover, we do not expect to see the second KK level states at energies not far above these TeV particles.

The existence of a stable weakly-interacting neutral particle, like the LTP, has important astrophysical implications. In this regard the LTP shares properties similar to the the lightest supersymmetric particle (LSP) in $R$-parity conserving supersymmetric Standard Model and the lightest KK-particle (LKP) in UED models. It can be a good dark matter candidate if it is neutral under the unbroken Standard Model gauge group. In the little Higgs model we proposed, the best candidates are the $B^{\prime}$ gauge boson and the $S U(2)_{W}$ singlets and neutral components of triplets in the scalar link fields. For $B^{\prime}$ LTP, it is similar to the case studied in the UEDs [11, 12]. The $B^{\prime}$ LTP gives the right relic density for dark matter if its mass is in the range of $600 \mathrm{GeV}-1.2 \mathrm{TeV}$ [38], which is consistent with the little Higgs model. The detection rates of $B^{\prime}$ LTP in various dark matter detection experiments are quite different from those of the LSP in the supersymmetric theories 39, 40, 41, 42, 43]. In particular, because the annihilation of two $B^{\prime}$ s into Standard Model fermions are not chirally suppressed, the indirect detection of $B^{\prime}$ LTPs annihilating into electron-positrons, 
neutrinos, and photons are much more promising than those of the LSP. For example, a peak in the positron energy distribution at the mass of $B^{\prime}$ may be seen in AMS, the anti-matter detector to be placed on the International Space Station, which is nonetheless not the case for the LSP [39].

As for scalar dark matter, it was recently studied in Ref. [44] for a different little Higgs model. In that model there is also an exact discrete symmetry, except that the heavy gauge bosons are neutral under that symmetry and the electroweak constraints are still a concern. It was found that there are two mass ranges for which the scalar LTP can give rise to the right relic density for dark matter: a low mass $\sim 100 \mathrm{GeV}$ if the LTP is mostly an $S U(2)_{W}$ singlet, and a high mass range $\gtrsim 500 \mathrm{GeV}$ if it is a mixture of the singlet and the neutral component of the triplet. In our case, the singlet annihilates through neither the weak gauge bosons nor the TeV gauge bosons, contrary to the case in Ref. [44], so it has to be even lighter than the low mass region in order to obtain the right relic density. On the other hand, the $S U(2)_{W}$ triplet scalars do interact with the light gauge bosons, and hence the estimate in Ref. [44] for the high mass range should roughly apply.

\section{CONCLUSIONS}

We have shown that, by proposing a symmetry acting only on new particles in the $\mathrm{TeV}$ scale, it is possible to relax the constraints, coming from the precision electroweak measurements, on the scale of new physics, thereby resolving the little hierarchy problem. The critical observation is that these constraints can be lowered by an order of magnitude if there is no tree-level exchanges of new heavy particles, which require interaction vertices containing only one new particle, among the Standard Model particles. On the other hand, stabilization of the electroweak scale necessitates cancellations of quadratic divergences to the Higgs mass-squared, which involve quantum loop diagrams and entail vertices with more than one new particles. Thus if one imposes a symmetry to eliminate the tree level exchanges of the heavy states, the electroweak scale can be stabilized naturally without conflicting with the precision measurements.

There are existing models with this kind of new symmetry in the TeV scale, for example the $R$-parity for supersymmetric theories and the KK-parity for UEDs. In this paper, we present a new model, in an entirely different class, with such a new TeV symmetry. It is a little Higgs model implemented with a $Z_{2}$ symmetry in the TeV scale which we call $T$-parity. This model has new particles at around $1 \mathrm{TeV}$, which are responsible for cutting off one-loop quadratic divergences, due to the Standard Model particles of the same spin, to the Higgs mass-squared and stabilizing the electroweak scale up to $10 \mathrm{TeV}$ without fine-tuning. At the same time, it is compatible with the precision data, a nice consequence of the $T$-parity. It is also an intriguing observation that all anomalies cancel in our model with the somewhat sophisticated $U(1)$ charge assignments.

The existence of this new $\mathrm{TeV}$ symmetry has many important implications for phenomenology, in addition to solving the little hierarchy problem. The lightest new particle charged under this TeV symmetry, the LTP, is a weakly-interacting stable particle. The LTP serves as a good candidate for dark matter if it is also neutral under the Standard Model gauge group, a property similar to its counterparts, the LSP in supersymmetric theories with $R$-parity and the LKP in UEDs. In terms of collider phenomenology, the typical signals are jets and/or leptons plus missing energies due to decays of $T$-odd heavy particles, which feature is shared by all three classes of theories: the supersymmetric theories with 
$R$-parity, UEDs with KK-parity, and little Higgs theories with $T$-parity. Nevertheless, more detailed studies can potentially tell these three categories of theories apart from one another.

As the completion date of the LHC approaches, the mystery of the TeV scale physics will be unraveled in the near future. Obviously it is of great interest to further explore the consequences of this new $\mathrm{TeV}$ symmetry in finer detail, whether they are specific to models or generic to theories with the new symmetry.

\section{Acknowledgments}

We thank N. Arkani-Hamed, P. Creminelli, M. Schmaltz, N. Toumbas, and J. Wacker for useful discussions. We also thank the Aspen Center for Physics for hospitality where part of this work was completed. This work is supported in part by the National Science Foundation under grant PHY-98-02709.

[1] S. Weinberg, "Phenomenological Lagrangians," PhysicaA 96, 327 (1979).

[2] W. Buchmuller and D. Wyler, "Effective Lagrangian Analysis Of New Interactions And Flavor Conservation," Nucl. Phys. B 268, 621 (1986).

[3] For a recent analysis, see R. Barbieri and A. Strumia, "What is the limit on the Higgs mass?," Phys. Lett. B 462, 144 (1999) arXiv:hep-ph/9905281.

[4] N. Arkani-Hamed, A. G. Cohen and H. Georgi, "Electroweak symmetry breaking from dimensional deconstruction," Phys. Lett. B 513, 232 (2001) hep-ph/0105239.

[5] N. Arkani-Hamed, A. G. Cohen, T. Gregoire and J. G. Wacker, "Phenomenology of electroweak symmetry breaking from theory space," JHEP 0208, 020 (2002) arXiv:hep-ph/0202089.

[6] N. Arkani-Hamed, A. G. Cohen, E. Katz, A. E. Nelson, T. Gregoire and J. G. Wacker, "The minimal moose for a little Higgs," JHEP 0208, 021 (2002) arXiv:hep-ph/0206020.

[7] N. Arkani-Hamed, A. G. Cohen, E. Katz and A. E. Nelson, "The littlest Higgs," JHEP 0207, 034 (2002) arXiv:hep-ph/0206021.

[8] J. Wudka, "Natural and model-independent conditions for evading the limits on the scale of new physics," arXiv:hep-ph/0307339.

[9] N. Arkani-Hamed, H.-C. Cheng, B. A. Dobrescu and L. J. Hall, "Self-breaking of the standard model gauge symmetry," Phys. Rev. D 62, 096006 (2000) arXiv:hep-ph/0006238.

[10] T. Appelquist, H.-C. Cheng and B. A. Dobrescu, "Bounds on universal extra dimensions," Phys. Rev. D 64, 035002 (2001) arXiv:hep-ph/0012100.

[11] H.-C. Cheng, K. T. Matchev and M. Schmaltz, "Radiative corrections to Kaluza-Klein masses," Phys. Rev. D 66, 036005 (2002) hep-ph/0204342.

[12] H.-C. Cheng, K. T. Matchev and M. Schmaltz, "Bosonic supersymmetry? Getting fooled at the LHC," Phys. Rev. D 66, 056006 (2002) arXiv:hep-ph/0205314.

[13] T. Appelquist and H. U. Yee, "Universal extra dimensions and the Higgs boson mass," Phys. Rev. D 67, 055002 (2003) arXiv:hep-ph/0211023.

[14] For example, K. m. Cheung and G. Landsberg, "Kaluza-Klein states of the standard model gauge bosons: Constraints from high energy experiments," Phys. Rev. D 65, 076003 (2002) arXiv:hep-ph/0110346 and references therein for earlier work. 
[15] N. Arkani-Hamed, A. G. Cohen and H. Georgi, "(De)constructing dimensions," Phys. Rev. Lett. 86, 4757 (2001) hep-th/0104005.

[16] C. T. Hill, S. Pokorski and J. Wang, Phys. Rev. D 64, 105005 (2001) arXiv:hep-th/0104035.

[17] T. Gregoire and J. G. Wacker, "Mooses, topology and Higgs," JHEP 0208, 019 (2002) arXiv:hep-ph/0206023.

[18] I. Low, W. Skiba and D. Smith, "Little Higgses from an antisymmetric condensate," Phys. Rev. D 66, 072001 (2002) arXiv:hep-ph/0207243.

[19] D. E. Kaplan and M. Schmaltz, "The little Higgs from a simple group," arXiv:hep-ph/0302049

[20] S. Chang and J. G. Wacker, "Little Higgs and custodial SU(2)," arXiv:hep-ph/0303001.

[21] S. Chang, "A 'littlest Higgs' model with custodial SU(2) symmetry," arXiv:hep-ph/0306034.

[22] W. Skiba and J. Terning, "A simple model of two little Higgses," arXiv:hep-ph/0305302.

[23] H. Georgi and A. Pais, "Calculability And Naturalness In Gauge Theories," Phys. Rev. D 10, 539 (1974).

[24] H. Georgi and A. Pais, "Vacuum Symmetry And The Pseudogoldstone Phenomenon," Phys. Rev. D 12, 508 (1975).

[25] D. B. Kaplan and H. Georgi, "SU(2) X U(1) Breaking By Vacuum Misalignment," Phys. Lett. B 136, 183 (1984).

[26] D. B. Kaplan, H. Georgi and S. Dimopoulos, "Composite Higgs Scalars," Phys. Lett. B 136, 187 (1984).

[27] H. Georgi and D. B. Kaplan, "Composite Higgs And Custodial SU(2)," Phys. Lett. B 145, 216 (1984).

[28] H. Georgi, D. B. Kaplan and P. Galison, "Calculation Of The Composite Higgs Mass," Phys. Lett. B 143, 152 (1984).

[29] M. J. Dugan, H. Georgi and D. B. Kaplan, "Anatomy Of A Composite Higgs Model," Nucl. Phys. B 254, 299 (1985).

[30] J. L. Hewett, F. J. Petriello and T. G. Rizzo, "Constraining the littlest Higgs," arXiv:hep-ph/0211218.

[31] C. Csaki, J. Hubisz, G. D. Kribs, P. Meade and J. Terning, "Big corrections from a little Higgs," Phys. Rev. D 67, 115002 (2003) arXiv:hep-ph/0211124.

[32] C. Csaki, J. Hubisz, G. D. Kribs, P. Meade and J. Terning, "Variations of little Higgs models and their electroweak constraints," arXiv:hep-ph/0303236.

[33] T. Gregoire, D. R. Smith and J. G. Wacker, "What precision electroweak physics says about the $\mathrm{SU}(6) / \mathrm{Sp}(6)$ little Higgs," arXiv:hep-ph/0305275.

[34] G. Burdman, M. Perelstein and A. Pierce, "Collider tests of the little Higgs model," Phys. Rev. Lett. 90, 241802 (2003) arXiv:hep-ph/0212228.

[35] T. Han, H. E. Logan, B. McElrath and L. T. Wang, "Phenomenology of the little Higgs model," Phys. Rev. D 67, 095004 (2003) arXiv:hep-ph/0301040.

[36] Z. Sullivan, "How to rule out little Higgs (and constrain many other models) at the LHC," arXiv:hep-ph/0306266.

[37] T. Han, H. E. Logan, B. McElrath and L. T. Wang, "Loop induced decays of the little Higgs: $\mathrm{H} \rightarrow$ g g, gamma gamma," Phys. Lett. B 563, 191 (2003) arXiv:hep-ph/0302188.

[38] G. Servant and T. M. Tait, "Is the lightest Kaluza-Klein particle a viable dark matter candidate?," Nucl. Phys. B 650, 391 (2003) arXiv:hep-ph/0206071.

[39] H.-C. Cheng, J. L. Feng and K. T. Matchev, "Kaluza-Klein dark matter," Phys. Rev. Lett. 89, 211301 (2002) arXiv:hep-ph/0207125.

[40] D. Hooper and G. D. Kribs, "Probing Kaluza-Klein dark matter with neutrino telescopes," 
Phys. Rev. D 67, 055003 (2003) arXiv:hep-ph/0208261.

[41] G. Servant and T. M. Tait, "Elastic scattering and direct detection of Kaluza-Klein dark matter," New J. Phys. 4, 99 (2002) arXiv:hep-ph/0209262.

[42] G. Bertone, G. Servant and G. Sigl, "Indirect detection of Kaluza-Klein dark matter," arXiv:hep-ph/0211342,

[43] D. Majumdar, "Detection rates for Kaluza-Klein dark matter," Phys. Rev. D 67, 095010 (2003) arXiv:hep-ph/0209277.

[44] A. Birkedal-Hansen and J. G. Wacker, "Scalar dark matter from theory space," arXiv:hep-ph/0306161. 